\title{
Geometric phase accumulation-based effects in the quantum dynamics of an anisotropically trapped ion
}

\author{
M. Scala, B. Militello and A. Messina \\ INFM, MIUR and Dipartimento di Scienze Fisiche ed Astronomiche \\ dell'Università degli Studi di Palermo, via Archirafi 36, I-90123 Palermo, Italy
}

\begin{abstract}
New physical effects in the dynamics of an ion confined in an anisotropic two-dimensional Paul trap are reported. The link between the occurrence of such manifestations and the accumulation of geometric phase stemming from the intrinsic or controlled lack of symmetry in the trap is brought to light. The possibility of observing in laboratory these anisotropy-based phenomena is briefly discussed.
\end{abstract}

keywords: Berry phase, Ion Traps

PACS: 39.10.+j, 03.65.Vf

\section{Introduction}

The continuous development of sophisticated techniques of cooling and trapping atoms witnessed over the last years provides physical systems ideal to test fundamental aspects of quantum mechanics. In particular ions confined in a Paul trap, an appropriately designed configuration of oscillating electromagnetic fields, exhibit a centre of mass (c.m.) motion describable as that of a quantum harmonic oscillator [1, 2, 3. With the help of a classical laser field it is possible to induce in the ion couplings between its vibrational and electronic degrees of freedom representable by more or less simple spin-boson hamiltonian models. When the laser wavelength is much larger than the amplitude of the ion c.m. oscillations (Lamb-Dicke regime) and the frequency of the classical field is tuned to one of the vibrational sidebands of the electronic transition under scrutiny (two-level approximation), the effective interaction may be described by Jaynes-Cummings-like models [4, 5, 6, 7, 8,

\footnotetext{
*email:matteo.scala@fisica.unipa.it
} 
The use of ion traps instead of cavities to test the dynamics originating from hamiltonian models of this kind has the advantage that environment effects on the ion may be reasonably neglected [2].

In this paper our attention is focused on anisotropic two-dimensional Paul traps, that is characterized by different oscillation frequencies along the principal axes of the trap. Our scope is to bring to light effects which are directly traceable back to such a lack of symmetry in the trap. When the ion is driven by a laser beam not collinear with any of the axes of the trap, we show that, unlike what happens in the isotropic case, there is no way to describe the system dynamics in terms of a time-independent interaction picture hamiltonian. We instead prove the existence of a simple and physically transparent resonance condition under which the interaction picture hamiltonian becomes sinusoidally oscillating at the difference between the two free oscillation frequencies of the c.m. of the ion. This implies that when the anisotropy is small enough the system dynamics may be faced with using the adiabatic approximation approach. Therefore the system we are going to study proves to be an ideal candidate to seek effects stemming from the Berry or geometric phase accumulation [9, 10].

The main result reported in this paper is twofold. On the one hand we show that anisotropy can be considered as a valuable resource to test the theory of adiabatic evolutions and non-dynamical phases.

On the other hand we put into evidence that the feasibility of experiments for measuring the Berry phase provides a way to reveal and quantitatively appreciate the presence of anisotropy in the trap.

The paper is organized as follows. In the next section we describe the physical system under study and in the third section its time-dependent effective hamiltonian under the action of an appropriate laser field is obtained. In the fourth section we analyze the adiabatic evolution of the physical system and calculate the Berry phase acquired by the instantaneous eigenstates of the hamiltonian. The fifth section is devoted to physical effects directly attributable to geometric phase accumulation. Finally in the sixth section some conclusive remarks are given.

\section{Ions in a Paul trap}

A Paul trap is a configuration of electrodes which generates a quadrupolar electromagnetic potential $\Phi(t)$ that, using cylindrical coordinates $r, \phi, z$, can be written as [1, 2, 3,

$$
\Phi(t)=\Phi_{0}(t) \frac{\left(r^{2}-2 z^{2}\right)}{2 r_{0}^{2}}
$$

where $r_{0}$ is a length parameter (trap radius) depending on the dimensions of the trap and $\Phi_{0}(t)$ introduces an appropriate time dependence.

If $\Phi_{0}(t)=U$ is constant, i.e. if the field generated by the electrodes is electrostatic, we cannot obtain any confinement for a charged particle moving in such a field, as well known from classical electromagnetic field theory. 
Confinement of a charged particle may be easily achieved by superposing to the static potential $U$ an oscillating one having amplitude $V$ and frequency $\Omega$ :

$$
\Phi_{0}(t)=U-V \cos (\Omega t)
$$

In this case, putting

$$
a_{z}=-2 a_{r}=-\frac{8 e U}{m r_{0}^{2} \Omega^{2}}, \quad q_{z}=-2 q_{r}=-\frac{4 e V}{m r_{0}^{2} \Omega^{2}}, \quad \zeta=\frac{\Omega t}{2}
$$

the equations of motion for a particle with charge $q$ and mass $m$ admit stable solutions, i.e. confinement for the charged particle, if the values of the parameters $a_{z}, q_{z}$, belong to appropriate zones of the space of parameters called stability zones [1]. Moreover, in this case it is possible to separate the charged particle motion into the sum of two components, one of which, called micromotion, is a very rapid oscillation with an amplitude small enough to be negligible with respect to the main component, the secular motion. The latter is a harmonic motion corresponding to an effective three-dimensional harmonic potential whose frequencies are given by [1]:

$$
\omega_{r}^{2}=\left(a_{r}+\frac{q_{r}^{2}}{2}\right) \frac{\Omega^{2}}{4}, \quad \omega_{z}^{2}=\left(a_{z}+\frac{q_{z}^{2}}{2}\right) \frac{\Omega^{2}}{4}
$$

and with which we can replace the real time-dependent potential given by eqs.(1) and (2).

It is important to emphasize that if we choose $U$ and $V$ in such a way that

$$
U=\frac{e V^{2}}{m r_{0}^{2} \Omega^{2}}
$$

we obtain $\omega_{r}=\omega_{z}$ and so the quadrupolar potential corresponding to these values gives rise to an effective potential which is spherically symmetric. Thus, starting from values of $U$ and $V$ related in accordance with eq. (5), changing at will the static potential $U$ allows the introduction of anisotropy in a prefixed axial plane (for instance $x-z$ ) of the Paul trap.

In particular we claim that current technology is compatible with the possibility of designing and realizing small Paul traps wherein a weak fully controllable anisotropy may be established.

Of course even an imperfect construction of the electrodes, for instance a bad or moderately good realization of their required geometric properties, may lead to an experimental setup operating in non-ideal conditions modelizable in terms of anisotropy, for example in the radial plane $(x-y)$.

\section{Physical system and hamiltonian model}

Let us consider a two level ion of mass $m$ confined in a two-dimensional $x-z$ Paul trap, whose oscillation frequencies are $\nu_{a}$ and $\nu_{b}$ respectively, interacting 
with a laser beam oriented along a direction which makes an angle $\alpha$ with the $x$ axis. It has been shown that this system, in the rotating wave approximation, may be described by the following hamiltonian model [4]:

$$
H_{R W A}=\hbar\left(\nu_{a} a^{\dagger} a+\nu_{b} b^{\dagger} b\right)+\frac{\hbar \omega_{0}}{2} \sigma_{z}+\left[d \epsilon^{(-)}(x, z, t) \sigma_{-}+h . c .\right]
$$

where $d$ is the appropriate dipole transition matrix element, $\hbar \omega_{0}$ the energy separation between the ground $(|-\rangle)$ and the excited $(|+\rangle)$ electronic states of the ion and $\sigma_{z}=|+\rangle\langle+|-|-\rangle\left\langle-\left|, \sigma_{+}=\right|+\right\rangle\langle-|$and $\sigma_{-}=|-\rangle\langle+|$.

The quantity $\epsilon^{(-)}(x, z, t)$ is the negative frequency part of the classical driving field, given by:

$$
\epsilon^{(-)}(x, z, t)=E_{0} \mathrm{e}^{-i[k(x \cos \alpha+z \sin \alpha)-\omega t]}
$$

where $\omega$ and $k$ are the frequency and the modulus of the wave vector of the laser respectively.

In a frame rotating at the laser frequency, the hamiltonian can be cast in the form $H=H_{t r a p}+H_{i n t}$, with:

$$
\left\{\begin{array}{l}
H_{\text {trap }}=\hbar\left(\nu_{a} a^{\dagger} a+\nu_{b} b^{\dagger} b\right)+\hbar \frac{\delta}{2} \sigma_{z} \\
H_{\text {int }}=\hbar \Omega\left\{e^{-i k(x \cos \alpha+z \sin \alpha)} \sigma_{-}+e^{i k(x \cos \alpha+z \sin \alpha)} \sigma_{+}\right\}
\end{array}\right.
$$

where $\delta=\omega_{0}-\omega$ is the detuning of the laser beam from the electronic transition frequency, $\Omega=\frac{d E_{0}}{\hbar}$ and $a\left(a^{\dagger}\right)$ and $b\left(b^{\dagger}\right)$ are the annihilation (creation) operators along the directions $x$ and $z$ respectively.

In the interaction picture with respect to the hamiltonian $H_{\text {trap }}$ given by (8), the exponentials appearing in $H_{\text {int }}$ can be expanded to obtain a sum of terms which oscillate at frequencies which are linear combinations of $\nu_{a}, \nu_{b}$ and $\delta$ with integer coefficients.

In the Lamb-Dicke limit $k \Delta x \cos \alpha, k \Delta z \sin \alpha<<1$ (where $\Delta x=\sqrt{\frac{\hbar}{2 m \nu_{a}}}$ and $\left.\Delta z=\sqrt{\frac{\hbar}{2 m \nu_{b}}}\right)$, under the simple resonance condition $\delta=\nu_{a}+\nu_{b}$ and assuming $\Delta \nu \equiv \nu_{a}-\nu_{b} \ll \min \left\{\nu_{a}, \nu_{b}\right\}$, the only terms which are both non negligible and slowly oscillating are those proportional to $a^{\dagger^{2}} \sigma_{-}, b^{\dagger^{2}} \sigma_{-}, a^{\dagger} b^{\dagger} \sigma_{-}$ and their hermitian conjugates. Performing the rotating wave approximation, also taking into account that $\Omega \ll \min \left\{\nu_{a}, \nu_{b}\right\}$, yields the following interaction picture hamiltonian

$$
H_{i n t}^{I}(t) \approx-\hbar \lambda\left[\left(\cos \theta a^{\dagger} \mathrm{e}^{-i \frac{\Delta \nu}{2} t}+\sin \theta b^{\dagger} \mathrm{e}^{i \frac{\Delta \nu}{2} t}\right)^{2} \sigma_{-}+h . c .\right]
$$

where

$$
\left\{\begin{array}{l}
\cos \theta=\frac{\Delta x \cos \alpha}{\sqrt{(\Delta x \cos \alpha)^{2}+(\Delta z \sin \alpha)^{2}}}, \quad \sin \theta=\frac{\Delta z \sin \alpha}{\sqrt{(\Delta x \cos \alpha)^{2}+(\Delta z \sin \alpha)^{2}}}, \\
\lambda=\frac{\Omega}{2} \mathrm{e}^{k^{2}\left(\Delta x^{2} \cos ^{2} \alpha+\Delta z^{2} \sin ^{2} \alpha\right)}\left[(\Delta x \cos \alpha)^{2}+(\Delta z \sin \alpha)^{2}\right] .
\end{array}\right.
$$


The quantity $\Delta \nu$ measures the anisotropy degree characterizing the vibrational ion dynamics.

The physically transparent result we obtain is the periodically time-dependent hamiltonian $H_{\text {int }}^{I}(t)$, following from the chosen resonance condition. This periodicity suggests that the system under scrutiny could be advantageously exploited to investigate effects related to Berry phase. It is worth noting that, had we chosen a different value of $\delta$, we would anyway have obtained a timedependent hamiltonian, which means that such an explicit temporal dependence in $H_{\text {int }}^{I}(t)$, periodic or not, entirely stems from the presence of anisotropy in the trap.

The anisotropy in the system under scrutiny gives thus rise at resonance to an intrinsic periodicity of the hamiltonian and to a possible accumulation of Berry phase in a period. With intrinsic we mean that periodicity and Berry phase do not appear as due to the manipulation of appropriate parameters, as, on the contrary, we see in the well known case of spin $1 / 2$ in an uniform varying magnetic field [9], but to the impossibility to find a condition on the laser frequency such to wash out any temporal dependence in the interaction picture hamiltonian.

In other words we conclude that the lack of symmetry of the trap is at the origin of the appearance of Berry phase accumulation in the system dynamics.

\section{Berry phase accumulation in a cycle}

It is well known that when the hamiltonian $H(t)$ of a physical system is slowly varying with time, its dynamical behaviour may be investigated with the help of the so-called adiabatic approximation [11. Assuming $H(t)$ possesses a nondegenerate discrete spectrum at any arbitrarily fixed $t$, the time evolution of the $n$-th eigenstate $|n(0)\rangle$ of $H(0)$ generates, at the time instant $t$, the $n$-th eigenstate $|n(t)\rangle$ of $H(t)$ [11. The anisotropy degree $\Delta \nu$ provides a useful reference frequency to quantitatively estimate the rapidity of variation of the instantaneous eigenstates of $H(t)$. Berry showed that there is a simple way to express the phase acquired by $|n(t)\rangle$ when, after some time $T$, the hamiltonian comes back to its initial form, i.e. $H(T)=H(0)$.

Assume that the hamiltonian of the system depends on a set of parameters $\mathbf{R}(t)=\left(R_{1}(t), R_{2}(t), \ldots\right)$, i.e. $H(t) \equiv H(\mathbf{R}(t))$, and call $\left\{E_{n}(\mathbf{R})\right\}$ the eigenvalues of $H(\mathbf{R})$ and $\{|n(\mathbf{R})\rangle\}$ the corresponding eigenstates. The evolution of the hamiltonian $H(t)$ corresponds to a curve in the space of parameters and the condition $H(T)=H(0)$ becomes $\mathbf{R}(T)=\mathbf{R}(0)$.

If the initial state of the system is $|\Psi(0)\rangle=|n(0)\rangle$, the state of the system at the time instant $T$ may be written down as $[9$ :

$$
|\Psi(T)\rangle=\mathrm{e}^{i \gamma_{n}^{C}} \mathrm{e}^{-\frac{i}{\hbar} \int_{0}^{T} d t^{\prime} E_{n}\left(\mathbf{R}\left(t^{\prime}\right)\right)}|\Psi(0)\rangle
$$

where the exponential factor containing the integral symbol, called dynamical phase factor, reduces to the usual phase factor in the case of time-independent 
hamiltonian. The quantity $\gamma_{n}^{C}$ is given by:

$$
\gamma_{n}^{C}=i \oint_{C}\left\langle n(\mathbf{R}) \mid \nabla_{R} n(\mathbf{R})\right\rangle \cdot \mathbf{d} \mathbf{R}
$$

the curve $C$ being the path followed by $\mathbf{R}(t)$ in the space of parameters. The quantity $\gamma_{n}^{C}$ is called Berry phase or geometric phase, since its value depends only on the curve $C$. Putting $\phi=\Delta \nu t / 2$, the hamiltonian (9) may be cast in the following form:

$$
H_{\phi}=-\hbar \lambda\left[A_{\phi}^{\dagger} \sigma_{-}+A_{\phi}^{2} \sigma_{+}\right]
$$

where $A_{\phi}^{\dagger}=\cos \theta \mathrm{e}^{-i \phi} a^{\dagger}+\sin \theta \mathrm{e}^{i \phi} b^{\dagger}$.

It is easy to verify that the creation operator $B_{\phi}^{\dagger}=\sin \theta \mathrm{e}^{i \phi} a^{\dagger}+\cos \theta \mathrm{e}^{-i \phi} b^{\dagger}$ corresponds to a vibrational mode orthogonal to that associated to $A_{\phi}$.

Let us introduce the bimodal Fock states

$$
|N\rangle_{\phi} \equiv|N\rangle_{A_{\phi}}|0\rangle_{B_{\phi}}=\frac{A_{\phi}^{\dagger}}{\sqrt{N !}}|0\rangle_{a}|0\rangle_{b}
$$

$|0\rangle_{a}\left(|0\rangle_{b}\right)$ being the vacuum state of the vibrational mode associated to $a(b)$. It is immediate to check directly using eq. (13) that the states $|0\rangle_{\phi}|-\rangle$ and $|1\rangle_{\phi}|-\rangle$ are degenerate eigenstates of $H_{\phi}$ with vanishing energy. The other eigenstates of $H_{\phi}$ are singlets of energy

$$
E_{N, \pm}= \pm \hbar \lambda \sqrt{N(N-1)} .
$$

and can be cast in the following form:

$$
\left|\Psi_{N \pm}(\phi)\right\rangle=\frac{1}{\sqrt{2}}\left(|N\rangle_{\phi}|-\rangle \mp|N-2\rangle_{\phi}|+\rangle\right)
$$

provided $N \geq 2$.

We see from eq. (15) that the coupling constant $\lambda$, at any time instant $t$, is of the order of the lowest Bohr frequency associated to the instantaneous spectrum of $H(t)$.

Denoting by $U(t)$ the evolution operator relative to $H_{\phi}$, the dynamics of the instantaneous eigenstates (16) after a cycle, in view of eq. (11), is given by

$$
U(T)\left|\Psi_{N \pm}(\phi=0)\right\rangle=\mathrm{e}^{-i \frac{E_{N, \pm}}{\hbar} T} \mathrm{e}^{i \gamma_{N \pm}(T)}\left|\Psi_{N \pm}(\phi=0)\right\rangle
$$

The associated geometrical phase $\gamma_{N \pm}(T)$ can be calculated by performing the integration in eq. (12) along the curve given by $\mathbf{R}=(\cos \phi, \sin \phi)$ and varying $\phi$ from 0 to $2 \pi$, obtaining the following explicit expression:

$$
\gamma_{N-}(T)=\gamma_{N+}(T) \equiv \gamma_{N}(T)=-2 \pi\left(2 \sin ^{2} \theta-1\right) N
$$

apart from terms independent from $N$, here omitted since they do not give rise to observable effects. 
The dependence of Berry phase on $N$ is the key building block for the result we are going to present in the next section: it indeed suggests that its occurrence may be revealed by preparing the system in a superposition of Fock states with different values of $N$.

Before closing this section we wish to write down a condition for the validity of the adiabatic approximation in the case under scrutiny. The evolution of the system can be considered as adiabatic if its anisotropy degree is sufficiently small with respect to the effective interaction strength $\lambda$. More quantitatively, the square modulus of the ratio between the frequency $\Delta \nu$, measuring the maximum rapidity of variation of the set $\{|n(t)\rangle\}$ of the instantaneous eigenstates of $H(t)$, and the minimum Bohr frequency appearing in the instantaneous spectrum of $H(t)$ has to be much smaller than unity [1]. In our case this condition becomes:

$$
|\Delta \nu|^{2} \ll|\lambda|^{2}
$$

A quantitative estimation of the applicability of inequality (19) in our situation will be done in the last section.

\section{Anisotropy vs. Berry phase effects}

In accordance with eq. (18) we now elaborate our strategy aimed at finding some physical effects directly traceable back to Berry phase accumulation and consequently to the presence of anisotropy in a two-dimensional Paul trap.

Firstly we choose an initial state quite simple in structure, experimentally feasible and appropriate to reveal Berry phase accumulation. In view of eq. (18), the quantum superposition of two different eigenstates of $H(0)$ accumulating different Berry phases meets our request. After this step we have to imagine a suitable physically transparent observable sensitive to the non-dynamical phase acquired by the eigenstates of $H(0)$ appearing in the initial state expansion.

To this end we propose to start with the following superposition

$$
|\Psi(t=0)\rangle=\frac{1}{\sqrt{2}}\left(|N\rangle_{\phi=0}+|N+1\rangle_{\phi=0}\right)|-\rangle
$$

which may be prepared by methods similar to that used to prepare Fock states in the experiments described in 12 .

In view of eqs. (16)-(17) and (18) the evolution of the state given by eq. 
) under $H_{\phi}$ may be cast in the following form:

$$
\begin{aligned}
& |\Psi(t=T)\rangle=\frac{1}{\sqrt{2}}\left(U(T)|N\rangle_{\phi=0}|-\rangle+U(T)|N+1\rangle_{\phi=0}|-\rangle\right)= \\
& =\frac{1}{\sqrt{2}}\left\{\mathrm{e}^{i \gamma_{N}(T)}\left[\cos \left(\frac{E_{N,+}}{\hbar} T\right)|N\rangle_{\phi=0}|-\rangle+i \sin \left(\frac{E_{N,+}}{\hbar} T\right)|N-2\rangle_{\phi=0}|+\rangle\right]+\right. \\
& \left.+\mathrm{e}^{i \gamma_{N+1}(T)}\left[\cos \left(\frac{E_{N+1,+}}{\hbar} T\right)|N+1\rangle_{\phi=0}|-\rangle+i \sin \left(\frac{E_{N+1,+}}{\hbar}\right)|N-1\rangle_{\phi=0}|+\rangle\right]\right\}
\end{aligned}
$$

As expected by construction, this state turns out to be the sum of two states having different geometrical phases.

In accordance with our strategy, we must look for an observable sensitive to Berry phase accumulation. A simple choice is represented by the operator

$$
\hat{O}=\frac{A_{\phi=0}^{\dagger}+A_{\phi=0}}{2}
$$

since it connects the two states of the superposition (21). This operator has a clear physical meaning, being proportional to the position operator of the vibrational mode corresponding to $\phi=0$.

If we choose the direction $\alpha$ of the laser such that $\theta=\pi / 6$, we obtain the following mean value after a cycle

$$
\begin{aligned}
& \left\langle\Psi(t=T)\left|\hat{O}_{I}\right| \Psi(t=T)\right\rangle=\frac{1}{2}\left\{\cos \left(\left[\gamma_{N}(T)-\gamma_{N+1}(T)\right]+\nu T\right) \times\right. \\
& \times\left[\cos \left(\frac{E_{N,+}}{\hbar} T\right) \cos \left(\frac{E_{N+1,+}}{\hbar} T\right) \sqrt{N+1}+\right. \\
& \left.\left.+\sin \left(\frac{E_{N,+}}{\hbar} T\right) \sin \left(\frac{E_{N+1,+}}{\hbar} T\right) \sqrt{N-1}\right]\right\}
\end{aligned}
$$

where $\hat{O}_{I}$ is the observable $\hat{O}$ in the interaction picture and $\nu=\frac{\nu_{a}+\nu_{b}}{2}$.

In view of eq. (18) and in correspondence of $\theta=\pi / 6$ we get

$$
\gamma_{N}(T)-\gamma_{N+1}(T)=\pi
$$

It thus turns out that the mean value of $\hat{O}$ given by eq. (23) is the negative of what we would obtain if the only phase factor acquired after a cycle by an eigenstate of the hamiltonian $H_{\phi}$ were the dynamical one. Taking $N$ large enough, and for an appropriate value of $\lambda$, the quantity between square brackets is much larger than unity and the difference in sign we have found can be considered as macroscopic. 
Moreover, let us consider the instantaneous energy eigenvalues given by eq. (15). Since they are independent of time, their structure is the same both in the case under scrutiny and in the case of an ion trapped in an isotropic trap $\left(\nu_{a}=\nu_{b}\right)$ and interacting with a laser beam oriented along the direction $\theta$ and with a detuning $\delta$ equal to the frequency of the trap. Thus we can say that in both cases (isotropic and anisotropic) one finds the same dynamical phases and that the only difference in the system dynamics is the appearance of the geometric phase factor in the case of an anisotropic trap.

Summing up, we claim the following result: the macroscopic difference in sign we have found is a direct manifestation of anisotropy. It allows us to distinguish at a certain time instant $T$ between an isotropic trap and an anisotropic one having oscillation frequencies such that $\Delta \nu=4 \pi / T$.

Such a behaviour also reflects the different structures of the states of the ion at the time instant $T$. Indeed from eqs. (21) and (24) it is possible to show that the states reached in the two cases (isotropic and anisotropic) are superpositions of the same couple of orthogonal states: the difference between an anisotropic trap and an isotropic one consists in the quantum phase difference of the two states in the superposition. It is of particular relevance that the states of the ion at the time instant $T$ in the two situations are orthogonal.

\section{Conclusive remarks}

In this paper we have investigated the quantum dynamics of an ion confined in a two-dimensional Paul trap characterized by a difference $\Delta \nu$ between the oscillation frequencies along its two principal axes. We have shown that when a controllable or intrinsic anisotropy is weak, a laser beam appropriately oriented gives rise to a vibronic coupling representable, in the interaction picture, by a periodic hamiltonian of frequency $\Delta \nu$.

If the coupling strength compared with $\Delta \nu$ makes it legitimate studying the dynamics of the system under the adiabatic approximation, we find a $|n(0)\rangle$ dependent and trap anisotropy-based Berry phase accumulation after a cycle. On the contrary we show that the associated dynamical phase is completely insensitive to the assumed lack of symmetry of the trap. We thus succeed in proposing simple and realizable appropriate initial conditions which after a cycle do possess quantum coherences transparently related to the non dynamical phase accumulation and then to the anisotropy of the trap.

The main result of this paper is the idea of exploiting such a deep geometricaldynamical connection to propose, at least in principle, a scheme to estimate quantitatively and qualitatively the absence of symmetry in the trap.

To this end we present a motivated choice of a simple and physically transparent observable whose mean value manifests macroscopic differences if referred to an isotropic or not oscillator.

Our analysis does not incorporate from the very beginning possible sources of decoherence, and thus we wish to conclude discussing the possibility of observing the reported anisotropy effects in laboratory. 
Condition (19) for the adiabaticity of the time evolution of the system imposes limitations to the square modulus of the ratio between the anisotropy degree $\Delta \nu$ and the coupling constant $\lambda$. Assuming accordingly $|\Delta \nu / \lambda|^{2} \approx 0,1$ immediately yields $\Delta \nu_{\max } \approx \lambda / 3$.

In experimental works on ions in Paul traps 12, testing an interaction described by a Jaynes-Cummings hamiltonian, it has been seen that the effects of decoherence effectively appear after about ten Rabi oscillations, i.e. the coherence time $\tau$ of the ion vibrational motion is about one tenth of the coupling constant of the interaction hamiltonian. If we suppose that this relation is also valid in the experimental scheme proposed by us, i.e. $1 / \tau \sim \lambda / 10$, the time $T=4 \pi / \Delta \nu$ taken to complete an adiabatic cyclic evolution is of the order of $\tau$. This means that, in such a scenario, possible decoherence effects would be still weak enough after a cycle to be negligible within a first approximation.

\section{References}

[1] P. K. Ghosh, Ion Traps, Clarendon Press, Oxford (1995)

[2] D. Wineland et al., J. Res. Natl. Inst. Stand. Technol. 103, 258 (1998)

[3] D. Leibfried et al., Rev. Mod. Phys., 75, 281 (2003)

[4] W. Vogel, D.-G. Welsch, S Wallentowitz, Quantum Optics: an Introduction, Wiley-Vch (2001)

[5] W. Vogel and R.L.de Matos Filho, Phys. Rev. A, 52, 4214 (1995)

[6] A. Messina et al., J. Mod. Opt., 50, 1-49 (2003)

[7] S. Maniscalco et al., Phys. Rev. A, 61, 053806 (2000)

[8] B. Militello et al., Phys. Rev. A, 66, 23402 (2002)

[9] M. V. Berry, Proc. R. Soc. Lond. A, 392, 45-57 (1984)

[10] M. V. Berry, J. Mod. Opt., 34, 1401-1407 (1987)

[11] A. Messiah, Quantum Mechanics, John Wiley \& Sons, New York 1958

[12] D. M. Meekhof et al., Phys. Rev. Lett., 76, 1796 (1996)

[13] D. Wineland and W.M. Itano, Phys. Rev. A, 20, 1521 (1979) 